\title{
Frequency and Antimicrobial Resistance Pattern among Bacterial Clinical Isolates Recovered from Different Specimens in Egypt
}

\author{
Rania Ibrahim Shebl ${ }^{1, *}$, Yasser Omar Mosaad ${ }^{2}$ \\ ${ }^{1}$ Microbiology and Immunology Department, Faculty of Pharmacy, Ahram Canadian University, Cairo, Egypt \\ ${ }^{2}$ Pharmacology, Toxicology and Biochemistry Department, Faculty of Pharmaceutical Sciences \& Pharmaceutical Industries, Future \\ University in Egypt, Cairo, Egypt
}

Email address:

Shbel.rania@gmail.com (R. I. Shebl),yasour_r@yahoo.co.uk(Y. O. Mosaad)

${ }^{*}$ Corresponding author

\section{To cite this article:}

Rania Ibrahim Shebl, Yasser Omar Mosaad. Frequency and Antimicrobial Resistance Pattern among Bacterial Clinical Isolates Recovered from Different Specimens in Egypt. Central African Journal of Public Health. Vol. 5, No.1, 2019, pp. 36-45.

doi: 10.11648/j.cajph.20190501.16

Received: December 5, 2018; Accepted: January 2, 2019; Published: January 29, 2019

\begin{abstract}
Antimicrobial resistance (AMR) is a global public health threat resulting in high mortality rates. Current study aimed to identify the most prevalent pathogens among variable infection sites and their AMR pattern. Data concerning cultures and antibiotic susceptibilities were retrieved from Microbiology Department's records and statistically analyzed. Out of 554 bacterial isolates, Gram negative isolates (68.4\%) were predominant. Urine specimens showed the highest incidence of recovery of total isolates $(41.5 \%, \mathrm{n}=230)$ followed by blood $(23.1 \%, \mathrm{n}=128)$, while sputum specimens exhibited the least frequency $(17 \%, \mathrm{n}=94)$. E. coli $(30.7 \%, \mathrm{n}=170)$, . aureus $(21.1 \%, \mathrm{n}=117)$ and Klebsiella spp $(20.9 \%$, n=116) were the most frequently isolated pathogens. Recovery of isolates was significantly more frequent among males $(\mathrm{P}<0.05)$ except in case of urine specimens. Highest incidence of resistance in both Gram positive and Gram negative isolates was recorded in case of cephalosporins and penicillin/ $\beta$-lactamase. Gram positive isolates exhibited the least resistance to linezolid (10.8\%) and vancomycin (9.5\%) whereas colistin was the most effective against Gram negative isolates as it recorded $16.4 \%$ resistance. Higher frequency of multiple drug resistance (MDR) was also observed in Gram negative isolates compared to Gram positive ones. Resistance to uropathogens and MDR were significantly more frequent in males. Although $E$. coli was the most prevalent uropathogen but it showed the least incidence of MDR however Pseudomonas spp exhibited the highest MDR rate. The high incidence of resistance in the current study is alarming and highlights the necessity of routinely monitoring the local prevalence of resistance for selecting the best antimicrobial treatment and as a guide for empirical therapy.
\end{abstract}

Keywords: Prevalence, Multiple Drug Resistance, Pattern, Egypt

\section{Introduction}

Emergence of antimicrobial resistance to different antibiotics is a critical problem that leads to a real danger of post antibiotic era [1]. During the last decade, many reports have documented the doubling or even tripling in the resistance rates of nearly all groups of serious pathogens [2] in addition to the progressive emergence of MDR isolates [3]. The lack of proper and early identification of the causative pathogens especially in patients with serious infection led to the administration of broad spectrum antibiotics. Such issue resulted in dramatic emergence of resistant strains that the magnified the problem of resistance [1]. The Center for Disease Control and Prevention (CDC) reported that high rates of infection with resistant pathogens all over the world resulted in passive influence on the global economy, loss in productivity and elevated death rates [4]. Data concerning the endemic antimicrobial resistance are usually unavailable especially in the areas of the world where antibiotics are available over the counter [5]. Despite that many reports demonstrated the incidence and the resistance patterns of many pathogens, few studies are available to 
estimate the endemic antimicrobial resistance profile in low and middle income countries [6]. Thus an evidence based knowledge regarding the local antimicrobial resistance pattern is considered an essential guide for treatment of specific pathogens as well as for empirical antimicrobial therapy [5]. This guide is also of significant importance in the implementation of the effective antimicrobial stewardship [1] as well as in the design of national and international research programs [5]. Therefore, the present study aimed to identify the microbial spectrum and the antimicrobial resistance pattern of the most prevalent pathogens recovered from variable infection sites in addition to determination of the prevalence of multiple drug resistance.

\section{Materials and Methods}

\subsection{Study Design}

Retrospective study was conducted utilizing the microbiology laboratory records of in-patients in an Egyptian hospital in Cairo. Microbiology records were reviewed and records showing mixed cultures or unidentified microorganisms as well as duplicate records for the same patients were excluded [7]. Records for specimens other than blood, sputum, urine and wound specimens were also excluded. Information regarding the identified bacterial isolate, specimen type, patient's gender and antimicrobial susceptibility was collected and recorded.

\subsection{Identification of the Isolated Organism}

Sample processing, identification of the microorganism to the genus and/or species level was performed according to the standard operating procedures of the hospital in place. Briefly, bacterial isolates were identified based on morphological characters, Gram stain and confirmatory biochemical test. Gram positive bacteria were identified using catalase reaction, coagulase test as well as via testing the hemolytic activity on blood agar. Identification of Gramnegative bacteria was carried out through inoculation on MacConkey agar plates, followed by biochemical tests such as oxidase and urease tests.

\subsection{Antimicrobial Susceptibility}

Antimicrobial susceptibilities of the bacterial isolates were determined using Kirby-Bauer disk diffusion method using Mueller Hinton agar plates according to the Clinical Laboratory Standards Institute (CLSI) guidelines. The tested antimicrobial discs were routinely supplied from Oxoid and Bioanalyse.

\subsection{Multiple Drug Resistance (MDR)}

MDR isolates were identified according to the guidelines recommended by European Centre for Disease Prevention and Control (ECDC) and the CDC. MDR isolates were identified as isolates showing resistance to at least one antimicrobial agent in three or more antimicrobial classes [8].

\subsection{Statistical Analysis}

Data were presented as counts and percentage. Statistical analysis was performed using statistical package for social sciences (SPSS) computer software (version 25), IBM software, USA. Pearson Chi-square test was performed to identify the significant effect of each antibiotic on different isolates as well as the relation between gender and prevalence of different isolates. Chi-square and Fisher exact tests were used to test the association between gender and resistance to different antibiotics. Statistically significant difference was considered at $\mathrm{p}$ value $\leq 0.05$.

\section{Results}

\subsection{Antibiotic Resistance Pattern}

Among total isolates $(\mathrm{n}=554)$, Gram negative isolates $(68.4 \%, n=379)$ were more prevalent compared to Gram positive ones $(31.6 \%, \mathrm{n}=175)$. Urine specimens showed the highest frequency of recovery of total isolates $(41.5 \%$, $\mathrm{n}=230)$ followed by blood $(23.1 \%, \mathrm{n}=128)$, while sputum specimens exhibited the least percentage $(17 \%, \mathrm{n}=94)$ of recovery. E. coli $(30.7 \%, \mathrm{n}=170), S$. aureus $(21.1 \%, \mathrm{n}=117)$ and Klebsiella spp $(20.9 \%, \mathrm{n}=116)$ were the most frequently isolated pathogens.

Table 1. Frequency of the predominant Gram positive pathogens among different specimens.

\begin{tabular}{|c|c|c|c|c|c|c|c|c|c|c|c|c|c|c|c|}
\hline \multirow{2}{*}{ Isolates } & \multicolumn{3}{|l|}{ Blood } & \multicolumn{3}{|l|}{ Sputum } & \multicolumn{3}{|l|}{ Urine } & \multicolumn{3}{|l|}{ Wound } & \multicolumn{3}{|l|}{ Total } \\
\hline & $\%(n)$ & $\mathrm{M}$ & $\% \mathrm{~F}$ & $\%(n)$ & $\% \mathrm{M}$ & $\% \mathrm{~F}$ & $\%(n)$ & $\% \mathrm{M}$ & $\% \mathrm{~F}$ & $\%(n)$ & $\% \mathrm{M}$ & $\% \mathrm{~F}$ & $\%^{\mathrm{a}}$ & $\% \mathrm{M}$ & $\% \mathrm{~F}$ \\
\hline anren & $57.3(67$ & 71.6 & 28.4 & 111 & 76.9 & 23.1 & $13.7(16)$ & 43.8 & 56.2 & $17.9(21)$ & 66.7 & 33.3 & 66.9 & 67.5 & 32.5 \\
\hline $\begin{array}{l}\text { eptococcus spp } \\
=36 \text { ) }\end{array}$ & $30.6(11)$ & 54.5 & 45.5 & $19.4(7)$ & 85.7 & 14.3 & $41.7(15)$ & 46.7 & 53.3 & $8.3(3)$ & 66.7 & 33.3 & 20.6 & 58.3 & 41.7 \\
\hline $\operatorname{CoNS}(n=22)$ & 40.9 & . & 55.6 & & 60 & 40 & & 30 & Jo & & & $2 J$ & & 54.5 & 45.5 \\
\hline Total & $49.7(87)$ & $66.7^{*}$ & $33.3 *$ & $14.3(25)$ & $76^{*}$ & $24 *$ & $20(35)$ & 45.7 & 54.3 & $16(28)$ & $67.9^{*}$ & $32.1 *$ & $\mathrm{~N}=175$ & $64 *$ & $36^{*}$ \\
\hline
\end{tabular}

CoNS; coagulase-negative Staphylococcus, N; Total number of Gram positive isolates, \% (n); percentage of each isolate relative to the total number of isolates, \% M; Percentage of isolates recovered from males, \% F: Percentage of isolates recovered from females, $\%^{\mathrm{a}}$ : percentage of each isolate relative to N, *; Statistically significant difference between the incidence of recovery of isolates from males and females.

Regarding total Gram positive isolates, blood specimens is the major source for recovery of Gram positive isolates as it presented $49.7 \%$. S. aureus was the most common pathogen (66.9\%), followed by Streptococcus spp (20.6\%), while coagulase-negative Staphylococcus (CoNS) recorded a frequency of $12.6 \%$. Among different specimens, S. aureus $(57.3 \%)$ and CoNS $(40.9 \%)$ were most frequently recovered from blood. Meanwhile, Streptococcus spp exhibited the 
highest incidence of recovery from urine specimens (41.7\%). Gram positive isolates were more common in males $(64 \%)$ compared to females $(36 \%)$. Assessment of the recovery of these pathogens among blood, sputum and wound specimens followed the same pattern except that there was no statistically significant difference $(\mathrm{P}>0.05)$ between the frequency of recovery of uropathogens from females $(54.3 \%)$ compared to males $(45.7 \%)$ (Table 1$)$.

Gram negative isolates showed predominance of $E$. coli $(44.9 \%, \mathrm{n}=170)$ followed by Klebsiella spp $(30.6 \%, \mathrm{n}=116)$ and Pseudomonas spp $(10.6 \%, \mathrm{n}=40)$. On the other side, Proteus spp $(6.3 \%, \mathrm{n}=24)$, non-lactose fermenters (NLF) $(6.1 \%, \mathrm{n}=23)$ and Acinetobacter spp $(1.6 \%, \mathrm{n}=6)$ were less frequent. The majority of Gram negative isolates were recovered from urine $(51.5 \%, \mathrm{n}=195)$ with the predominance of E. coli $(58.2 \%, \mathrm{n}=99)$ and Klebsiella spp $(55.2 \%, \mathrm{n}=64)$. Wound and sputum specimens were also found as another source for recovery of E.coli, where they showed frequency of recovery in the order of $18.2 \%$ and $14.7 \%$, respectively. Pseudomonas spp also showed high incidence of recovery from urine $(40 \%, \mathrm{n}=16)$ followed by wound $(27.5 \%, \mathrm{n}=11)$. Recorded data revealed higher incidence of total Gram negative isolates $(60.7 \%)$ in male patients. Gram negative isolates in male patients was significantly $(\mathrm{P}<0.05)$ more common than females among different specimens except in case of uropathogens where the difference between the incidence of recovery of these isolates was statistically nonsignificant among male and female patients (Table 2).

Table 2. The spectrum of Gram negative isolates in different specimens.

\begin{tabular}{|c|c|c|c|c|c|c|c|c|c|c|c|c|c|c|c|}
\hline \multirow{2}{*}{ Isolates } & \multicolumn{3}{|l|}{ Blood } & \multicolumn{3}{|l|}{ Sputum } & \multicolumn{3}{|l|}{ Urine } & \multicolumn{3}{|l|}{ Wound } & \multicolumn{3}{|l|}{ Total } \\
\hline & $\%$ (n) & $\% \mathbf{M}$ & $\% \mathrm{~F}$ & $\%$ (n) & $\% \mathrm{M}$ & $\% \mathbf{F}$ & $\%(n)$ & $\% \mathrm{M}$ & $\% \mathrm{~F}$ & $\%$ (n) & $\% \mathbf{M}$ & $\% \mathrm{~F}$ & $\%^{a}$ & $\% \mathbf{M}$ & $\% \mathrm{~F}$ \\
\hline E. coli $(\mathrm{n}=170)$ & $8.8(15)$ & 73.3 & 26.7 & $14.7(25)$ & 88 & 12 & $58.2(99)$ & 39.4 & 60.6 & $18.2(31)$ & 90.3 & 9.7 & 44.9 & 58.8 & 41.2 \\
\hline $\begin{array}{l}\text { Pseudomonas spp } \\
(\mathrm{n}=40)\end{array}$ & $15(6)$ & 66.7 & 33.3 & $17.5(7)$ & 71.4 & 28.6 & $40(16)$ & 50 & 50 & $27.5(11)$ & 72.7 & 27.3 & 10.6 & 62.5 & 37.5 \\
\hline $\begin{array}{l}\text { Klebsiella spp } \\
(n=116)\end{array}$ & $13.8(16)$ & 50 & 50 & 20.7 (24) & 70.8 & 29.2 & $55.2(64)$ & 46.9 & 53.1 & $10.3(12)$ & 91.7 & 8.3 & 30.6 & 56.9 & 43.1 \\
\hline $\begin{array}{l}\text { Proteus spp } \\
(n=24)\end{array}$ & $4.2(1)$ & 100 & 0 & $12.5(3)$ & 100 & 0 & $16.7(4)$ & 75 & 25 & $66.7(16)$ & 75 & 25 & 6.3 & 79.2 & 20.8 \\
\hline $\operatorname{NLF}(n=23)$ & $4.3(1)$ & 100 & 0 & $34.8(8)$ & 50 & 50 & $47.8(11)$ & 72.7 & 27.3 & $13.0(3)$ & 100 & 0 & 6.1 & 69.6 & 30.4 \\
\hline $\begin{array}{l}\text { Acinetobacter } \\
\operatorname{spp}(n=6)\end{array}$ & $33.3(2)$ & 100 & 0 & $33.3(2)$ & 50 & 50 & $16.7(1)$ & 0 & 100 & $16.7(1)$ & 100 & 0 & 1.6 & 66.7 & 33.3 \\
\hline Total & $10.8(41)$ & $65.9 *$ & $34.1 *$ & $18.2(69)$ & $75.4 *$ & $24.6 *$ & $51.5(195)$ & 45.1 & 54.9 & $19.5(74)$ & $85.1 *$ & $14.9 *$ & $\mathrm{~N}=379$ & $60.7 *$ & $39.3^{*}$ \\
\hline
\end{tabular}

NLF; Non-lactose fermenters, N; Total number of Gram negative isolates, \% (n); percentage of each isolate relative to the total number of isolates, \% M; Percentage of isolates recovered from males, \% F: Percentage of isolates recovered from females, $\%{ }^{\mathrm{a}}$ : percentage of each isolate relative to $\mathrm{N}$, *; Statistically significant difference between the incidence of recovery of isolates from males and females.

\subsection{Antibiotic Resistance Pattern of Isolates Recovered from Various Infection Sites}

\subsubsection{Gram Positive Isolates}

Highest incidence of resistance was recorded to third generation cephalosporins especially in case of ceftazidime $(100 \%, \mathrm{n}=22)$ and cefixime $(97.9 \%, \mathrm{n}=48)$ with lower resistance to ceftriaxone $(56.3 \%, \mathrm{n}=135)$ and cefotaxime (44.4\%, $n=27)$. Whereas, cefoperazone showed the least resistance either alone $(27.8 \%, \mathrm{n}=18)$ or in combination with sulbactam $(25 \%, \mathrm{n}=72)$. High resistance rate was also recorded in case of first, second and fourth generation cephalosporins. The combination between penicillin and $\beta$ lactamase inhibitors exhibited high incidence of resistance especially in case of amoxicillin/clavulanic acid $(70.2 \%$, $\mathrm{n}=121)$ followed by ampicillin/sulbactam $(55.8 \%, \mathrm{n}=104)$. While piperacillin/tazobactam retained most of its antimicrobial activity where it showed only $25 \%$ resistance $(n=16)$. Regarding macrolides, azithromycin exhibited a resistance rate in the order of $57.1 \%(n=35)$. Clindamycin, fusidic acid and fluoroquinolones showed moderate resistance, but lower resistance was recorded in case of carbapenems and teicoplanin. On the other side, the least resistance was observed towards nitrofurantoin (11.8\%, $\mathrm{n}=34)$, linezolid $(10.8 \%, \mathrm{n}=65)$ and vancomycin $(9.5 \%$, $\mathrm{n}=84$ ) (Table 3).

Regarding different isolates, $S$. aureus exhibited high resistance rates to most antibiotics, whereas it showed lower resistance to vancomycin $(10.6 \%, \mathrm{n}=66)$, linezolid $(14 \%$, $\mathrm{n}=43)$, teicoplanin $(22.4 \%, \mathrm{n}=58)$ and cefoperazone/sulbactam $(20.8 \%, \mathrm{n}=48)$ in addition to $100 \%$ susceptibility to nitrofurantoin. Streptococcus spp and CoNS followed similar pattern but Streptococcus spp was $100 \%$ susceptible to both vancomycin $(n=10)$ and piperacillin/tazobactam $(n=2)$. Moreover, all CoNS isolates were susceptible to linezolid $(n=10)$, imipenem $(n=8)$ and piperacillin/tazobactam $(n=3)$. Data also revealed a statistically significant difference in the antimicrobial potentials to different isolates in case of amoxicillin/clavulanic acid, ceftriaxone, cefixime, cefoperazone/sulbactam and glycopeptide antibiotics (Table 3).

Table 3. Resistance profile among Gram positive isolates recovered from different specimens.

\begin{tabular}{|c|c|c|c|c|c|c|}
\hline \multirow{2}{*}{ Antimicrobial classes } & \multirow{2}{*}{ Antimicrobial agent } & \multicolumn{4}{|c|}{ \% Resistance (n) } & \multirow{2}{*}{ P value } \\
\hline & & S. aureus & Streptococci & CoNS & Total isolates & \\
\hline \multirow[t]{2}{*}{ 1-Penicillin/ $\beta$-lactamase inhibitors } & Amox/clavu & $76.8(82)$ & $69.2(26)$ & $30.8(13)$ & $70.2 *(121)$ & 0.03 \\
\hline & Amp/sulb & $53.7(67)$ & $54.5(22)$ & $66.7(15)$ & $55.8(104)$ & 0.21 \\
\hline
\end{tabular}




\begin{tabular}{|c|c|c|c|c|c|c|}
\hline \multirow{2}{*}{ Antimicrobial classes } & \multirow{2}{*}{ Antimicrobial agent } & \multicolumn{4}{|c|}{ \% Resistance (n) } & \multirow{2}{*}{ P value } \\
\hline & & S. aureus & Streptococci & CoNS & Total isolates & \\
\hline \multirow{4}{*}{ 2-Cephalosporin $1^{\text {st }} \mathrm{G}$} & Piper/tazob & $36.4(11)$ & $0(2)$ & $0(3)$ & $25(16)$ & 0.25 \\
\hline & Cefazolin & $63.6(11)$ & $0(1)$ & $66.7(3)$ & $60(15)$ & 0.40 \\
\hline & Cephradine & $78.6(14)$ & $80(5)$ & $50(2)$ & $76.2(21)$ & 0.7 \\
\hline & Cephalexin & $83.3(12)$ & $50(4)$ & $80(5)$ & $76.2(21)$ & 0.08 \\
\hline \multirow[t]{2}{*}{ Cephalosporin $2^{\text {nd }} \mathrm{G}$} & Cefuroxime & $42.2(45)$ & $46.2(13)$ & $25(8)$ & $40.9(66)$ & 0.22 \\
\hline & Cefaclor & $69.2(13)$ & $62.5(8)$ & $50(2)$ & $65.2(23)$ & 0.77 \\
\hline \multirow[t]{5}{*}{ Cephalosporin $3^{\text {rd }} \mathrm{G}$} & Ceftriaxone & $53.3(92)$ & $75(28)$ & $25(16)$ & $56.3 *(135)$ & 0.04 \\
\hline & Ceftazidime & $100(12)$ & $100(7)$ & $100(2)$ & $100(22)$ & - \\
\hline & Cefotaxime & $38.9(18)$ & $40(5)$ & $75(4)$ & $44.4(27)$ & 0.26 \\
\hline & Cefixime & $97.5(40)$ & $100(5)$ & $100(3)$ & $97.9 *(48)$ & 0.04 \\
\hline & Cefoperazone & $25(12)$ & $25(4)$ & $50(2)$ & $27.8(18)$ & 0.53 \\
\hline Cephalosporin $4^{\text {th }} \mathrm{G}$ & Cefepime & $83.3(12)$ & $55.6(9)$ & $60(5)$ & $69.2(26)$ & 0.18 \\
\hline 3. Cephalosporin/ $\beta$-lactamase inhibitors & Cefoperazone/Sulb & $20.8(48)$ & $31.3(16)$ & $37.5(8)$ & $25 *(72)$ & 0.03 \\
\hline \multirow[t]{2}{*}{ 4-Carbapenems } & Meropenem & $27.4(84)$ & $47.6(21)$ & $18.2(11)$ & $30.2(116)$ & 0.17 \\
\hline & Imipenem & $33.3(18)$ & $55.6(9)$ & $0(8)$ & $31.4(35)$ & 0.08 \\
\hline \multirow[t]{2}{*}{ 5-Glycopeptide antibiotics } & Teicoplanin & $22.4(58)$ & $66.7(12)$ & $16.7(12)$ & $28.0 *(82)$ & 0.02 \\
\hline & Vancomycin & $10.6(66)$ & $0(10)$ & $12.5(8)$ & $9.5 *(84)$ & 0.04 \\
\hline 6- Macrolide & Azithromycin & $63.6(22)$ & $44.4(9)$ & $50(4)$ & $57.1(35)$ & 0.07 \\
\hline 7- Oxazolidinones & Linezolid & $14.0(43)$ & $8.3(12)$ & $0(10)$ & $10.8(65)$ & 0.13 \\
\hline 8- Lincosamides & Clindamycin & $53.2(47)$ & $70(10)$ & $33.3(6)$ & $54.0(63)$ & 0.5 \\
\hline 9-Tetracycline & Doxycycline & $27.8(72)$ & $45.8(24)$ & $50(10)$ & $33.9(106)$ & 0.07 \\
\hline 10-Fusidane & Fusidic acid & $64.7(17)$ & $50(2)$ & $50(6)$ & $40(25)$ & 0.49 \\
\hline \multirow[t]{4}{*}{ 11- Fluoroquinolone } & Norfloxacin & $42.9(14)$ & $75(8)$ & NT & $54.2(22)$ & 0.17 \\
\hline & Ofloxacin & $33.3(15)$ & $50(10)$ & $75(4)$ & $44.8(29)$ & 0.14 \\
\hline & Ciprofloxacin & $50(14)$ & $37.5(8)$ & $66.7(6)$ & $50(28)$ & 0.76 \\
\hline & Levofloxacin & $37.8(37)$ & $25(8)$ & $66.7(9)$ & $40.7(54)$ & 0.06 \\
\hline 12- Nitrofurans & Nitrofurantoin & $0(14)$ & $20(15)$ & $20(5)$ & $11.8(34)$ & 0.17 \\
\hline
\end{tabular}

Amox/clavu; amoxicillin/clavulanic acid, Amp/sulb; ampicillin/sulbactam, Piper/tazob; piperacillin/tazobactam, G; generation, NT; not tested, n; number of bacterial isolates tested against each antimicrobial agent, *; statistically significant difference between the effect of each antibiotic on different isolates.

\subsubsection{Gram Negative Isolates}

Gram negative isolates exhibited high resistance rates to most antibiotic classes such as cephalosporins. However, the combination between cefoperazone and sulbactam reduced the resistance to cefoperazone from $77.2 \%$ to $42.4 \%$. Penicillin/ $\beta$ lactamase inhibitors also showed high degree of resistance especially in case of amoxicillin/clavulanic acid $(92.3 \%, n=78)$ followed by ampicillin/sulbactam $(71.7 \%, n=329)$, while the combination between piperacillin and tazobactam showed lower resistance $(57.2 \%, \mathrm{n}=173)$. Sulfamethoxazole either alone or in combination with trimethoprim resulted also in high resistance rates in the order of $85.7 \%$ and $88.8 \%$, respectively. High incidence of resistance was also recorded in case of azteronam $(81.1 \%, \mathrm{n}=90)$, azithromycin $(73.2 \%, \mathrm{n}=71)$ as well as towards fluoroquinolones. Similar pattern was also observed in case of gentamicin $(66.4 \%, n=122)$ and doxycycline $(62.8 \%, \mathrm{n}=121)$, imipenem $(44.9 \%, \mathrm{n}=78)$ and meropenem $(38 \%, \mathrm{n}=284)$ (Table 4$)$.

Nitrofurantoin showed potential antimicrobial activity against $E$. coli, where the percentage of resistance against it was $17.2 \%$. Meanwhile, Klebsiella spp, NLF, Pseudomonas spp and Proteus spp recorded high resistance rates to nitrofurantoin in the order of $60.7 \%, 77.8 \%, 92.9 \%$ and $100 \%$, respectively. Moreover, E. coli showed high resistance rates to most antimicrobial classes with lower resistance to piperacillin/tazobactam $(43.3 \%, \mathrm{n}=67)$ and amikacin $(34.6 \%$, $\mathrm{n}=104)$, followed by cefoperazone/sulbactam $(27.8 \%, \mathrm{n}=90)$ and meropenem $(23.3 \%, n=120)$. On the other side, the least resistance was observed in case of colistin $(6.7 \%, \mathrm{n}=15)$. Higher degree of resistance was recorded in case of Klebsiella spp compared to E. coli. Pseudomonas spp also followed similar resistance profile with the least resistance to both imipenem and colistin $(12.5 \%, \mathrm{n}=8)$. In addition, an elevated resistance in case of other bacterial isolates such as Proteus spp, Acinetobacter spp and NLF was also recorded. It was also obvious that, colistin was the most promising antimicrobial agent either against each Gram negative isolate or in case of total isolates, where it exhibited a resistance rate in the order of $16.4 \%$. Data also revealed that some antimicrobials showed a statistically significant difference in their antimicrobial activities to different bacterial isolates as presented in Table 4 .

Table 4. Resistance pattern in Gram negative isolates recovered from variable specimens.

\begin{tabular}{|c|c|c|c|c|c|c|c|c|c|}
\hline \multirow{2}{*}{ Antimicrobial classes } & \multirow{2}{*}{$\begin{array}{l}\text { Antimicrobial } \\
\text { agent }\end{array}$} & \multicolumn{6}{|c|}{ \% Resistance (n) } & \multirow{2}{*}{$\begin{array}{l}\text { Total } \\
\text { isolates }\end{array}$} & \multirow{2}{*}{ P value } \\
\hline & & E. coli & Klebsiella & Pseudomonas & Proteus & Acinetobacter & NLF & & \\
\hline \multirow{2}{*}{$\begin{array}{l}\text { 1-Penicillin/ } \beta \text {-lactamase } \\
\text { inhibitors }\end{array}$} & Amox/clavu & $81.8(33)$ & $100(22)$ & $100(11)$ & $100(3)$ & $100(2)$ & $100(7)$ & $92.3(78)$ & 0.14 \\
\hline & Amp/sulb & $62.5(144)$ & $82.5(103)$ & $81.8(33)$ & $80(25)$ & $50(6)$ & $61.1(18)$ & $71.7 *(329)$ & 0.006 \\
\hline
\end{tabular}




\begin{tabular}{|c|c|c|c|c|c|c|c|c|c|}
\hline \multirow{2}{*}{ Antimicrobial classes } & \multirow{2}{*}{$\begin{array}{l}\text { Antimicrobial } \\
\text { agent }\end{array}$} & \multicolumn{6}{|c|}{ \% Resistance (n) } & \multirow{2}{*}{$\begin{array}{l}\text { Total } \\
\text { isolates }\end{array}$} & \multirow{2}{*}{ P value } \\
\hline & & E. coli & Klebsiella & Pseudomonas & Proteus & Acinetobacter & NLF & & \\
\hline & Piper/tazob & $43.3(67)$ & $76(50)$ & $43.5(23)$ & $66.7(15)$ & $83.3(6)$ & $58.3(12)$ & $57.2 *(173)$ & 0.004 \\
\hline \multirow[t]{3}{*}{ 2-Cephalosporin $1^{\text {st }} \mathrm{G}$} & Cefazolin & $100(14)$ & $100(11)$ & $100(2)$ & $100(2)$ & NT & $100(1)$ & $100(30)$ & - \\
\hline & Cephradine & $81.5(27)$ & $81.3(16)$ & $100(4)$ & $100(3)$ & NT & $100(3)$ & $84.9(53)$ & 0.6 \\
\hline & Cephalexin & $92.9(14)$ & $100(22)$ & $83.3(6)$ & $100(6)$ & $100(1)$ & $100(2)$ & $96.1(51)$ & 0.84 \\
\hline \multirow{2}{*}{ Cephalosporin $2^{\text {nd }} \mathrm{G}$} & Cefuroxime & $64.7(17)$ & $100(13)$ & $83.3(6)$ & $100(1)$ & $100(1)$ & $100(2)$ & $82.5(40)$ & 0.15 \\
\hline & Cefaclor & $84.1(44)$ & $93.5(31)$ & $100(7)$ & $100(6)$ & $100(1)$ & $100(6)$ & $90.5(95)$ & 0.45 \\
\hline \multirow[t]{5}{*}{ Cephalosporin $3^{\text {rd }} \mathrm{G}$} & Ceftriaxone & $72.6(117)$ & $83.7(86)$ & $86.2(29)$ & $100(17)$ & $80(5)$ & $82.4(17)$ & $80.1 *(271)$ & 0.008 \\
\hline & Ceftazidime & $84.8(33)$ & 89.7 (29) & $66.7(12)$ & $100(5)$ & $100(3)$ & $71.4(7)$ & $84.3(89)$ & 0.27 \\
\hline & Cefotaxime & $63.5(52)$ & $87.1(31)$ & $61.5(13)$ & $60(5)$ & $100(2)$ & $100(5)$ & $72.2 *(108)$ & 0.02 \\
\hline & Cefixime & $78.3(23)$ & $100(17)$ & $100(11)$ & $83.3(6)$ & $100(2)$ & $100(6)$ & $90.8(65)$ & 0.15 \\
\hline & Cefoperazone & $78(50)$ & $84.8(33)$ & $47.1(17)$ & $85.7(7)$ & $100(3)$ & $100(4)$ & $77.2 *(114)$ & 0.04 \\
\hline Cephalosporin $4^{\text {th }} \mathrm{G}$ & Cefipeme & $80(20)$ & $93.8(16)$ & $75(8)$ & $85.7(7)$ & NT & $50(2)$ & $83(53)$ & 0.43 \\
\hline $\begin{array}{l}\text { 3. Cephalosporin/ } \beta \text { - } \\
\text { lactamase inhibitors }\end{array}$ & $\begin{array}{l}\text { Cefoperazone/ } \\
\text { Sulb }\end{array}$ & $27.8(90)$ & $62.9(62)$ & $41.2(17)$ & $46.7(15)$ & $100(1)$ & $38.5(13)$ & $42.4 *(198)$ & 0.001 \\
\hline 4- Monobactam & Aztreonam & $65.4(26)$ & $96.7(30)$ & $61.1(18)$ & $100(8)$ & $100(4)$ & $100(4)$ & $81.1 *(90)$ & 0.004 \\
\hline \multirow[t]{2}{*}{ 5-Carbapenems } & Meropenem & $23.3(120)$ & $40.4(89)$ & $51.7(29)$ & $51.7(21)$ & $83.3(6)$ & $63.2(19)$ & $38 *(284)$ & $<0.001$ \\
\hline & Imipenem & $39.3(28)$ & $48.4(31)$ & $12.5(8)$ & $83.3(6)$ & $50(2)$ & $66.7(3)$ & $44.9 *(78)$ & 0.048 \\
\hline \multirow[t]{2}{*}{ 6-Aminoglycosides } & Gentamicin & $48.8(43)$ & $65.9(41)$ & $87.5(16)$ & $81.8(11)$ & $100(3)$ & $87.5(8)$ & $66.4 *(122)$ & 0.04 \\
\hline & Amikacin & $34.6(104)$ & $57.3(82)$ & $37.1(35)$ & $60.9(23)$ & $57.1(7)$ & $52.9(17)$ & $45.9(268)$ & 0.12 \\
\hline 7- Macrolide & Azithromycin & $60.7(28)$ & $83.3(24)$ & $75(8)$ & $80(5)$ & $100(2)$ & $75(4)$ & $73.2 *(71)$ & 0.485 \\
\hline 8-Tetracycline & Doxycycline & $56.7(60)$ & $61.8(34)$ & $82.4(17)$ & $80(5)$ & $50(2)$ & $66.7(3)$ & $62.8(121)$ & 0.27 \\
\hline \multirow[t]{4}{*}{ 9- Fluoroquinolones } & Norfloxacin & $64.3(70)$ & $78.3(46)$ & $64.3(14)$ & $40(5)$ & NT & $91.7(12)$ & $70.1(147)$ & 0.11 \\
\hline & Ofloxacin & $63.6(55)$ & $72.1(43)$ & $76.5(17)$ & $66.7(9)$ & $50(2)$ & $100(9)$ & $70.4(135)$ & 0.33 \\
\hline & Ciprofloxacin & $63.6(77)$ & $70.7(58)$ & $50(16)$ & $64.3(14)$ & $100(3)$ & $70.6(17)$ & $65.9 *(185)$ & 0.004 \\
\hline & Levofloxacin & $66.7(78)$ & $82(50)$ & $54.5(22)$ & $80(15)$ & $100(3)$ & $77.8(9)$ & 71.8 (177) & 0.31 \\
\hline 10- Synthetic quinolone & Nalidixic acid & $75(12)$ & $85.7(7)$ & $100(3)$ & NT & $0(1)$ & $100(1)$ & $79.2(24)$ & 0.32 \\
\hline 11- Polymyxins & Colistin & $6.7(15)$ & $10.5(19)$ & $12.5(8)$ & NT & $0(2)$ & $25(4)$ & $16.4 *(55)$ & 0.03 \\
\hline \multirow[t]{2}{*}{ 12- Sulfonamide } & Sulfamethoxazole & $85(20)$ & $92.3(13)$ & NT & $100(1)$ & NT & $0(1)$ & $85.7(35)$ & 0.17 \\
\hline & Trimeth/Sulfa & $90.2(41)$ & $82.3(23)$ & $83.3(6)$ & $100(4)$ & $100(1)$ & $100(5)$ & $88.8(80)$ & 0.66 \\
\hline 13- Nitrofurans & Nitrofurantoin & $17.2(87)$ & $60.7(61)$ & $92.9(14)$ & $100(6)$ & $0(1)$ & $77.8(9)$ & $43.8 *(178)$ & $<0.001$ \\
\hline
\end{tabular}

Amox/clavu; amoxicillin/clavulanic acid, Amp/sulb; ampicillin/sulbactam, Piper/tazob; piperacillin/tazobactam, Trimeth/Sulfa; trimethoprim/sulfamethoxazole, G; generation, NT; not tested, n; number of bacterial isolates tested against each antimicrobial agent, *; Statistically significant difference between the effect of each antibiotic on different isolates.

\subsection{Multiple Drug Resistance}

Recorded data revealed that MDR occurs in $58.9 \%$ of total Gram positive isolates with a significant prevalence of MDR in males (66\%). S. aureus exhibited the highest incidence of MDR (59.8\%), followed by Streptococcus spp (58.3\%) and CoNS (54.5\%). MDR was more common in blood $(60.9 \%$, $\mathrm{n}=87)$ and urine $(62.9 \%, \mathrm{n}=35)$ isolates, with lower frequency in wound $(50 \%, \mathrm{n}=28)$ and sputum $(56 \%, \mathrm{n}=25)$. Isolates recovered from blood, sputum and wound showed also a significant higher frequency of MDR among male patients, except in case of urine isolates where there was no significant difference between the prevalence of MDR among male and female patients (Table 5).

Table 5. Multiple drug resistance pattern in Gram positive isolates.

\begin{tabular}{llllllllllllllll}
\hline Bacterial isolates & Blood & \multicolumn{3}{c}{ Sputum } & \multicolumn{3}{c}{ Urine } & \multicolumn{3}{c}{ Wound } & \multicolumn{3}{c}{ Total } \\
\hline & \% MDR & \% M & \% F & \% MDR & \% M & \% F & \% MDR & \% M & \% F & \% MDR & \% M & \% F & \% MDR & \% M & \% F \\
\hline S. aureus & 59.7 & 72.5 & 27.5 & 76.9 & 80 & 20 & 62.5 & 50 & 50 & 47.6 & 63.6 & 36.4 & 59.8 & 68.6 & 31.4 \\
Streptococcus spp & 72.7 & 62.5 & 37.5 & 28.6 & 100 & 0 & 60 & 55.6 & 44.4 & 66.7 & 50 & 50 & 58.3 & 61.9 & 38.1 \\
CoNS & 55.6 & 40.0 & 60.0 & 40 & 100 & 0 & 75 & 33.3 & 66.7 & 50 & 100 & 0 & 54.5 & 58.3 & 41.7 \\
Total & 60.9 & $67.9 *$ & $32.1 *$ & 56 & $85.7 *$ & $14.3 *$ & 62.9 & 50 & 50 & 50 & $68.8 *$ & $31.3 *$ & 58.9 & $66.0 *$ & $34.0 *$ \\
\hline
\end{tabular}

CoNS; coagulase-negative Staphylococcus, *; Statistically significant difference between the incidence of MDR among male and female patients.

Higher incidence of MDR $(77 \%)$ was recorded in Gram negative isolates compared to Gram positive ones. E. coli showed the least percentage of MDR (67.6\%), while Pseudomonas spp exhibited the highest incidence of MDR (95\%). A significant higher frequency of MDR was observed in males $(63 \%)$ compared to females $(37 \%)$. Blood, sputum and wound isolates exhibited similar profile but the difference between the incidence of MDR in male and female uropathogens was non-significant ( $p>0.05$ ) (Table 6). 
Table 6. Multiple drug resistance profile among Gram negative isolates.

\begin{tabular}{|c|c|c|c|c|c|c|c|c|c|c|c|c|c|c|c|}
\hline Bacterial isolates & Blood & & & Sputum & & & Urine & & & Wound & & & Total & & \\
\hline & $\%$ MDR & $\% \mathbf{M}$ & $\% \mathbf{F}$ & $\%$ MDR & $\% \mathbf{M}$ & $\% \mathbf{F}$ & $\%$ MDR & $\% \mathbf{M}$ & $\%$ F & $\%$ MDR & $\% \mathbf{M}$ & $\% \mathbf{F}$ & $\%$ MDR & $\% \mathbf{M}$ & $\% \mathbf{F}$ \\
\hline E. coli & 80 & 75 & 25 & 68 & 94.1 & 5.9 & 67.7 & 44.8 & 55.2 & 61.3 & 89.5 & 10.5 & 67.6 & 62.6 & 37.4 \\
\hline Pseudomonas spp & 66.7 & 75 & 25 & 100 & 71.4 & 28.6 & 100 & 50.0 & 50 & 100 & 72.7 & 27.3 & 95 & 63.2 & 36.8 \\
\hline Klebsiella spp & 75 & 41.7 & 58.3 & 91.7 & 72.7 & 27.3 & 79.7 & 51.0 & 49 & 75 & 88.9 & 11.1 & 81 & 58.5 & 41.5 \\
\hline Proteus spp & 100 & 100 & 0 & 100 & 100 & 0 & 100 & 75.0 & 25 & 81.3 & 69.2 & 30.8 & 87.5 & 76.2 & 23.8 \\
\hline NLF & 100 & 100 & 0 & 100 & 50 & 50 & 81.8 & 77.8 & 22.2 & 33.3 & 100 & 0.0 & 82.6 & 68.4 & 31.6 \\
\hline Total & 78 & $65.6^{*}$ & $34.4^{*}$ & 85.5 & $76.3^{*}$ & $23.7^{*}$ & 75.4 & 50.3 & 49.7 & 73 & $81.5^{*}$ & $18.5^{*}$ & 77 & $63 *$ & $37 *$ \\
\hline
\end{tabular}

NLF; Non-lactose fermenters, *; Statistically significant difference between the frequency of MDR among male and female patients

\subsection{Prevalence of Resistance Among Uropathogens}

Higher incidence of resistance to most antibiotics was significantly observed in isolates that were recovered from males compared to females (Table 7).

Table 7. Antibiotic resistance pattern in Gram positive and Gram negative uropathogens relative to gender.

\begin{tabular}{|c|c|c|c|c|c|c|c|c|}
\hline \multirow[b]{3}{*}{ Antimicrobial agent } & \multicolumn{8}{|c|}{ \% Resistance } \\
\hline & \multicolumn{4}{|c|}{ Gram positive } & \multicolumn{4}{|c|}{ Gram negative } \\
\hline & Male & Female & Total no. & $P$ value & Male & Female & Total no. & $P$ value \\
\hline Amox/clavu & 75 & 36.4 & 23 & 0.098 & 88.9 & 85.7 & 46 & 1 \\
\hline Amp/sulb & 71.4 & 36.4 & 18 & 0.367 & $75.7 *$ & $61 *$ & 170 & 0.018 \\
\hline Piper/tazob & 0 & 0 & 2 & - & $53.8^{*}$ & $25^{*}$ & 50 & 0.019 \\
\hline Cefazolin & 100 & - & 1 & 1 & 100 & 100 & 13 & - \\
\hline Cephradine & 66.7 & 100 & 8 & 0.375 & $100 *$ & $64.7^{*}$ & 34 & 0.02 \\
\hline Cephalexin & 60 & 66.7 & 8 & 1 & 100 & 93.8 & 29 & 1 \\
\hline Cefuroxime & $75^{*}$ & $0 *$ & 10 & 0.033 & 80 & 75 & 22 & 1 \\
\hline Cefaclor & 75 & 50 & 8 & 1 & 90.5 & 84.8 & 54 & 0.691 \\
\hline Ceftriaxone & $90 *$ & $50 *$ & 22 & 0.019 & $83.1 *$ & $61.6^{*}$ & 132 & 0.002 \\
\hline Ceftazidime & 100 & 100 & 8 & - & 66.7 & 77.8 & 27 & 0.609 \\
\hline Cefotaxime & 50 & 50 & 4 & 1 & $85.2 *$ & $40.9 *$ & 49 & $<0.001$ \\
\hline Cefixime & 100 & - & 1 & 1 & 100 & 100 & 9 & - \\
\hline Cefoperazone & 100 & - & 1 & 1 & 89.7 & 75 & 57 & 0.089 \\
\hline Cefepime & 100 & 100 & 2 & - & 71.4 & 85.7 & 14 & 1 \\
\hline Cefoperazone/sulb & $42.9 *$ & $9.1^{*}$ & 18 & 0.046 & $45.2 *$ & $19.6^{*}$ & 98 & 0.003 \\
\hline Meropenem & $60 *$ & $25 *$ & 22 & 0.045 & $30.1 *$ & $10 *$ & 143 & $<0.001$ \\
\hline Imipenem & 33.3 & 0 & 4 & 1 & 33.3 & 22.2 & 33 & 0.491 \\
\hline Aztreonam & - & - & - & - & 84.6 & 75 & 21 & 1 \\
\hline Vancomycin & 0 & - & 1 & - & - & - & - & - \\
\hline Azithromycin & - & - & - & - & 100 & 28.6 & 8 & 0.167 \\
\hline Gentamicin & - & - & - & - & 60 & 50 & 25 & 0.569 \\
\hline Linezolid & $0 \%$ & - & 2 & - & - & - & - & - \\
\hline Clindamycin & 100 & 100 & 2 & - & - & - & - & - \\
\hline Doxycycline & 33.3 & 55.6 & 21 & 0.161 & 64.9 & 56.3 & 69 & 0.41 \\
\hline Amikacin & - & - & - & - & $41.5 *$ & $20 *$ & 103 & 0.012 \\
\hline Norfloxacin & 62.5 & 45.5 & 19 & 0.387 & $79.7 *$ & $56.1^{*}$ & 125 & 0.004 \\
\hline Ofloxacin & 40 & 14.3 & 12 & 0.559 & 72.2 & 60.5 & 74 & 0.109 \\
\hline Ciprofloxacin & 50 & 42.9 & 11 & 1 & $68.1^{*}$ & $54.9^{*}$ & 95 & 0.025 \\
\hline Levofloxacin & 0 & 33.3 & 11 & 0.491 & $77.1 *$ & $57.5^{*}$ & 75 & 0.050 \\
\hline Nitrofurantoin & 7.7 & 10 & 33 & 1 & $54.7^{*}$ & $34^{*}$ & 175 & 0.002 \\
\hline Nalidixic acid & - & - & - & - & 100 & 75 & 21 & 0.113 \\
\hline Colistin & - & - & - & - & 0 & 20 & 8 & 0.385 \\
\hline Sulphamethoxazole & - & - & - & - & 91.7 & 84.2 & 31 & 1 \\
\hline Trimeth/Sulfa & - & - & - & - & 87.1 & 88.1 & 73 & 0.98 \\
\hline
\end{tabular}

Amox/clavu; amoxicillin/clavulanic acid, Amp/sulb; ampicillin/sulbactam, Piper/tazob; piperacillin/tazobactam, Trimeth/Sulfa; trimethoprim/sulfamethoxazole, n; number of Gram positive bacterial isolates, *; statistically significant difference between male and female isolates.

\section{Discussion}

Evaluating the altitudes of the problem of AMR is a challenge as the levels of antimicrobial resistance vary among healthcare settings and geographical regions. Infections with MDR pathogens result in postponed therapy which causes negative impact on the patient's health especially in case of immunocompromised individuals [9]. Moreover, adequate recognition of the proper use of antibiotics in each 
community is a key factor in the progress of resistance [10]. Current study aimed to determine the most predominant pathogens in our community and their antimicrobial resistance pattern.

In the present study, urinary tract infection was the most prevalent followed by blood stream infection with least frequency in case of respiratory tract infection. Gram negative isolates were mostly involved in urinary tract infections while Gram positive isolates were responsible for blood stream infection (BSI). Resembling our findings, a study reported that all the recovered uropathogens were Gram negative whereas $60 \%$ of the isolates causing BSI were Gram positive with highest incidence of $S$. aureus [2]. In the mean context, it was reported that urine specimens contributes in the recovery of $55.2 \%$ of bacterial isolates whereas blood, wound and sputum cultures were responsible for $25.3 \%, 16.2 \%$, and $3.3 \%$ of isolates, respectively [11]. Moreover, a study demonstrated that Gram negative isolates were more common $(61.3 \%, \mathrm{n}=57)$ with the predominance of E. coli $(\mathrm{n}=36)$ [2]. S. aureus $(22.8 \%, \mathrm{n}=100)$, Klebsiella pneumoniae $(14.8 \%, \mathrm{n}=65)$ and E. coli $(9.3 \%, \mathrm{n}=41)$ were also reported as the most common pathogens among variable specimens in another study [12]. In agreement with the current study E. coli and Klebsiella pneumoniae weren't only the most frequently isolated pathogens among Gram negative isolates [13] but they also represented the most predominant pathogens relative to other uropathogens [14-16].

Although many studies reported that E. coli was as the most predominant isolate recovered from urine specimens but on the contrary to our results Klebsiella spp was categorized as the sixth most common uropathogen in one study [17] and S. aureus was the second pathogen involved in urinary tract infection (UTI) in another study [18]. The similarities as well as the variation in the type and frequency of these pathogens among different studies could be related to many factors such as environmental conditions, health practices, patient conditions, personal hygiene, number of patients involved in each study and laboratory procedures [19].

E.coli is not only one of the major pathogens responsible for UTI but it also plays a key role in wound and respiratory tract infection. Similar to current findings, E. coli was the most frequently isolated from urine specimens $(85.9 \%)$ followed by wound $(8.4 \%)$ and sputum specimens $(5.6 \%)$ [20].

In the same context to the current results, Pseudomonas spp was one of the most prevalent Gram negative pathogens associated with urinary tract infections as well as in surgical sites [21]. In addition to other studies which reported that pus is the major source from which pseudomonas could be recovered [10, 22].

Regarding BSI, the current data highlights the participation of Gram positive pathogens in this type of infection with higher rate of recovery of Gram positive isolates by about 2 folds compared to Gram negative isolates. Whereas the incidence of MDR among BSI was higher in Gram negative isolates compared to Gram positive ones. That was also supported by a study which demonstrated that among BSI, $59 \%$ of bacterial isolates were Gram positive however the frequency of MDR in Gram positive isolates was low $(19.4 \%)$ compared to that in case of Gram negative isolates $(34.2 \%)$ [23].

It is also important to point out the involvement of $S$. aureus and CoNS in BSI where both pathogens were reported as the most frequently isolated from blood specimens [17, 12], respectively. Despite that our study revealed the superior contribution of $S$. aureus in the occurrence of BSI compared to CoNS. Another study demonstrated that among Gram positive isolates participating in BSI, CoNS $(38.8 \%, \mathrm{n}=72)$ was the most common pathogen followed by $S$. aureus $(20.8 \%)$ [23].

Concerning the antimicrobial resistance pattern, the effectiveness of vancomycin against $S$. aureus was obvious in the current study in addition to other studies [11]. For example, it was reported that all $S$. aureus isolates were $100 \%$ susceptible to vancomycin [24].

In coincidence with the present study, higher incidence of resistance was recorded in Gram negative isolates compared to Gram positive ones [10]. In addition, E. coli demonstrated elevated resistance rates to ciprofloxacin and third generation cephalosporins compared to lower resistance towards nitrofurantoin [12]. In another study, E. coli exhibited elevated resistance to nalidixic acid and ceftriaxone [20]. Also in a study carried out in Mansoura University Hospitals (Cairo), it was observed that E. coli was highly resistant to cefuroxime $(96 \%)$, ceftriaxone $(92 \%)$, cefaclor $(90 \%)$ and ciprofloxacin $(76 \%)$ whereas lower resistance was recorded against meropenem (40\%), imipenem (30\%) and amikacin (16\%) [25]. Also in agreement with our results, it was reported that $E$. coli exhibited the lowest percentage of MDR despite that it was the predominant uropathogen [18].

Resembling our findings, resistance to $\beta$-lactam antibiotics was reported as a major problem in a study carried out by Ibrahim and Hameed [13]. But on the contrary to the present study, they demonstrated lower resistance levels of Gram negative bacteria to amikacin, gentamicin and doxycycline in addition to high sensitivity of Gram positive isolates to macrolides and clindamycin [10]. The variation in the resistance pattern between the current study and other studies indicates this profile is influenced by variable determinants such as the diversity among different geographical regions [23], time during which each study was carried out as well as the study population [11].

Regarding resistance profile of Pseudomonas species and in agreement with the present study an elevated resistance rate was recorded against piperacillin/tazobactam and cefipime whereas higher sensitivity was observed to amikacin in addition to maximum sensitivity to imipenem [26]. Despite that another study reported that Pseudomonas aeruginosa was most commonly isolated from male patients, but it showed no resistance either to imipenem or colistin. The same study pointed out low antimicrobial resistance towards ceftazidime, piperacillin/tazobactam and cefipime [22], but these records weren't consistent with the current 
findings. This may be attributed to the variation between the detected pathogens in both studies and may indicate emergence of resistance in our community.

The recorded high incidence of MDR among Pseudomonas spp may be justified by the reported selective pressure due to mutations in chromosomal genes that led to production of extended spectrum $\beta$-lactamases (ESBL) as well as hyper expression of AmpC gene and the role of the efflux pumps. In addition to another resistance mechanism which is mediated through horizontal transfer of transposable elements that are coding for metallo- $\beta$-lactamases. Pseudomonas spp may also gain resistance to antibiotics as a consequence of interference with antibiotic permeability to the cell surface due to biofilm formation [21].

Elevated incidence of resistance to third generation cephalosporins and aztreonam as well as lower resistance rates to carbapenems in the present study might indicate the emergence of ESBL producing organisms in our community due to antibiotic abuse [10]. This is dependent on the fact that ESBLs are defined as Gram-negative bacteria that produce $\beta$ lactamases resulting in resistance to first, second and third generation cephalosporins as well as aztreonam whereas they aren't able to confer resistance to carbapenems. ESBLs are also antagonized by inhibitors of $\beta$-lactamase such as clavulanic acid [27]. This could justify the obvious decrease in resistance which was recorded in the current study when cefoperazone (third generation cephalosporin) was combined with sulbactam ( $\beta$-lactamase inhibitor) compared to the recorded elevated resistance against cefoperazone alone.

On the other side, the resistance to carbapenems may be related to efflux pumps and mutations in penicillin binding proteins. These mechanisms might enhance the resistance in case of Klebsiella pneumoniae, $P$. aeruginosa and Acinetobacter baumannii [28]. Thus the recorded higher resistance in case of Klebsiella spp compared to E. coli in the present study may be related to infections with Klebsiellaproducing carbapenemase-2 (KPC-2) or Metalloproteinase-1 producing $K$. pneumoniae [11].

Current study also recorded the emergence of resistance against colistin although it is considered the last line of defense against carbapenemase-producing Enterobacteriaceae. That might be attributed to the expression of plasmid-mediated colistin-resistant genes [29]. Also in consistence with our study, the bacterial uropathogens that were recovered from males showed higher incidence of resistance compared to females [18].

The rapid emergence of resistance is a global disaster that coincides with the regression in the discovery of new antibiotics [30]. It is worth to highlight that unreasonable consumption of antibiotics as well as transmission of resistant isolates among patients accounted for the progress in AMR rates [20]. Thus effective infection control measures [31], identification of the resistance mechanisms and the rational use of antibiotics through implementing effective antimicrobial stewardship are essential concerns. This stewardship should depend on assessment of the local prevalence of pathogens and their resistance profile so it could potentially manage the danger of AMR through reducing the selective pressure exerted on sensitive strains [32].

\section{Conclusions}

Gram negative isolates were more prevalent compared to Gram positive ones. Urinary tract infection was the most common followed by blood stream infection with highest incidence of E. coli, S. aureus and Klebsiella spp among total isolates. E. coli was the most common isolate accounting for urinary tract and wound infection whereas $S$. aureus was most frequently associated with blood stream infection. Males were more frequently subjected to different types of infections compared to females.

Highest incidence of resistance was associated with cephalosporins, followed by penicillin/ $\beta$-lactamase inhibitors. However Gram positive isolates exhibited the lowest resistance to linezolid and vancomycin whereas colistin was the most effective antimicrobial agent against Gram negative isolates. Despite that the discovery of nitrofurantoin isn't recent but it retained most of its potentials especially against $E$. coli as well as Gram positive isolates.

Elevated frequency of MDR was obvious among Gram negative isolates. Although E. coli was the most prevalent pathogen but it showed the least incidence of MDR. Contrarily, Pseudomonas spp exhibited the highest MDR rate. Prevalence of MDR was higher in males except in case of uropathogens. The elevated resistance rates in case of pathogens that were recovered from males reflect the necessity of considering the patient's gender in case of empirical prescription of antimicrobials. Also, the emerging resistance to carbapenems and colistin should also be taken into account and spot light on the importance of effective control measures.

It is necessary to note that antimicrobial therapy should take into account the data regarding the local prevalence of causative pathogens and their antimicrobial resistance profile rather than the universal guidelines. The present study presents a whole vision regarding the antimicrobial resistance pattern for the most frequent bacterial isolates among different specimens as well as essential considerations during empirical antimicrobial therapy. This local prevalence will also aid in establishing an effective antimicrobial stewardship to preserve the potentials of the current antimicrobial agents.

\section{Conflict of Interest}

The authors declare that they have no competing interests.

\section{Acknowledgements}

The authors extend their appreciation to Dr. Ahmed Shaltout (clinical pharmacy department) for his aid in data collection. 


\section{References}

[1] Akova M. (2016): Epidemiology of antimicrobial resistance in bloodstream infections. Virulence 7(3): 252-266.

[2] Paul R, Ray J, Sinha S, Mondal J 2017. Antibiotic resistance pattern of bacteria isolated from various clinical specimens: an eastern Indian study. International Journal of Community Medicine and Public Health 4(4): 1367-1371.

[3] Prestinaci F, Pezzotti P, Pantosti A 2015. Antimicrobial resistance: a global multifaceted phenomenon. Pathogens and global health 109(7): 309-318.

[4] Tom F 2013. Antibiotic resistance threats in the United States. Centres for Disease Control and Prevention. US Department of Health and Human Services. Available at: https://www.cdc.gov/drugresistance/pdf/ar-threats-2013508.pdf.

[5] El Kholy A, Baseem H, Hall GS, Procop GW, Longworth DL 2003. Antimicrobial resistance in Cairo, Egypt 1999-2000: a survey of five hospitals. Journal of Antimicrobial Chemotherapy 51(3): 625-630.

[6] Laxminarayan R, Duse A, Wattal C, Zaidi AK, Wertheim HF, Sumpradit N, Greko C 2013. Antibiotic resistance-the need for global solutions. The Lancet infectious diseases 13(12): 1057-1098.

[7] Pakyz AL 2007. The utility of hospital antibiograms as tools for guiding empiric therapy and tracking resistance: insights from the Society of Infectious Diseases Pharmacists. Pharmacotherapy: The Journal of Human Pharmacology and Drug Therapy 27(9): 1306-1312.

[8] Ansari S, Nepal HP, Gautam R, Shrestha S, Neopane P, Gurung G, Chapagain ML 2015. Community acquired multidrug resistant clinical isolates of Escherichia coli in a tertiary care center of Nepal. Antimicrobial resistance and infection control 4(1): 15 .

[9] Anderson DJ, Engemann JJ, Harrell LJ, Carmeli Y, Reller LB, Kaye KS 2006. Predictors of mortality in patients with bloodstream infection due to ceftazidime-resistant Klebsiella pneumoniae. Antimicrobial agents and chemotherapy 50(5): 1715-1720.

[10] Saravanan R, Raveendaran V 2013. Antimicrobial resistance pattern in a tertiary care hospital: an observational study. Journal of basic and clinical pharmacy 4(3): 56.

[11] Ntirenganya C, Manzi O, Muvunyi CM, Ogbuagu O 2015. High prevalence of antimicrobial resistance among common bacterial isolates in a tertiary healthcare facility in Rwanda. The American journal of tropical medicine and hygiene 92(4): 865-870.

[12] Moremi N, Claus H, Mshana SE 2016. Antimicrobial resistance pattern: a report of microbiological cultures at a tertiary hospital in Tanzania. BMC infectious diseases 16(1): 756.

[13] Ibrahim IA, Hameed TA 2015. Isolation, characterization and antimicrobial resistance patterns of lactose-fermenter enterobacteriaceae isolates from clinical and environmental samples. Open journal of medical microbiology 5(1): 169176.
[14] Behzadi P, Behzadi E, Yazdanbod H, Aghapour R, Cheshmeh MA, Omran DS 2010. A survey on urinary tract infections associated with the three most common uropathogenic bacteria. Maedica 5(2): 111.

[15] Beyene G, Tsegaye W 2011. Bacterial uropathogens in urinary tract infection and antibiotic susceptibility pattern in jimma university specialized hospital, southwest ethiopia. Ethiopian journal of health sciences 21(2): 141-146.

[16] Sajed AN, Batool U, Iram S, Yousaf NW, Asghar MN, Khan S 2014. Prevalence of urinary tract infections and their antibiotic sensitivity in tertiary care hospital Lahore. Journal of Dental and Medical Sciences 13(12): 57-61.

[17] Japoni A, Vazin A, Hamedi M, Davarpanah M. A, Alborzi A, Rafaatpour N 2009. Multidrug-resistant bacteria isolated from intensive-care-unit patient samples. Brazilian Journal of Infectious Diseases 13(2): 118-122.

[18] Linhares I, Raposo T, Rodrigues A, Almeida A 2013. Frequency and antimicrobial resistance patterns of bacteria implicated in community urinary tract infections: a ten-year surveillance study (2000-2009). BMC infectious diseases 13(1): 19.

[19] Mohammed MA, Alnour TM, Shakurfo OM, Aburass MM 2016. Prevalence and antimicrobial resistance pattern of bacterial strains isolated from patients with urinary tract infection in Messalata Central Hospital, Libya. Asian Pacific journal of tropical medicine 9(8): 771-6.

[20] Gautam R, Chapagain, ML, Acharya A, Rayamajhi N, Shrestha S, Ansari S, Nepal, HP 2013. Antimicrobial susceptibility patterns of Escherichia coli from various clinical sources. Journal of Chitwan Medical College 3(1): 14-17.

[21] Gupta R, Malik A, Rizvi M, Ahmed SM 2016. Incidence of multidrug-resistant pseudomonas spp. in ICU patients with special reference to ESBL, AMPC, MBL and biofilm production. Journal of global infectious diseases 8(1): 25 .

[22] Golia S, Suhani MS 2016. Isolation of Pseudomonas aeruginosa from various Clinical Isolates and its Antimicrobial Resistance Pattern in a Tertiary Care Hospital. Int. J. Curr. Microbiol. App. Sci 5(3): 247-253.

[23] Orsini J, Mainardi C, Muzylo E, Karki N, Cohen N, Sakoulas $G$ 2012. Microbiological profile of organisms causing bloodstream infection in critically ill patients. Journal of clinical medicine research 4(6): 371.

[24] Al-Zoubi MS, Al-Tayyar IA, Hussein E, Al Jabali A, Khudairat S 2015. Antimicrobial susceptibility pattern of Staphylococcus aureus isolated from clinical specimens in Northern area of Jordan. Iranian journal of microbiology 7(5): 265.

[25] Elsayed A, Mohamedin A, Ata T, Ghazala N 2016. Molecular Characterization of Multidrug Resistant Clinical Escherichia coli Isolates. Am J Bio Mol Biol 6:72-83.

[26] Fatima A, Naqvi SB, Khaliq SA, Perveen S, Jabeen S 2012. Antimicrobial susceptibility pattern of clinical isolates of Pseudomonas aeruginosa isolated from patients of lower respiratory tract infections. SpringerPlus 1(1): 70.

[27] Paterson DL, Bonomo RA 2005. Extended-spectrum $\beta$ lactamases: a clinical update. Clinical microbiology reviews 18(4): 657-686. 
[28] Papp-Wallace KM, Endimiani A, Taracila MA, Bonomo RA 2011. Carbapenems: past, present, and future. Antimicrobial agents and chemotherapy 55(11): 4943-4960.

[29] Wong SC, Tse H, Chen JH, Cheng VC, Ho PL, Yuen KY 2016. Colistin-resistant Enterobacteriaceae carrying the mcr-1 gene among patients in Hong Kong. Emerging infectious diseases 22(9): 1667.

[30] Ventola CL 2015. The antibiotic resistance crisis: part 1: causes and threats. Pharmacy and Therapeutics 40(4): 277.
[31] Uchil RR, Kohli GS, KateKhaye VM, Swami OC 2014. Strategies to combat antimicrobial resistance. Journal of clinical and diagnostic research 8(7): ME01.

[32] Karam G, Chastre J, Wilcox MH, Vincent JL 2016. Antibiotic strategies in the era of multidrug resistance. Critical Care 20(1): 136 . 